\title{
Gérontologie, nouveau paradigme
}

\section{Gerontology, new paradigm}

\author{
A. Franco \\ (C) Springer-Verlag France 2010
}

Le vieillissement de la planète devient palpable. Peut-être plus encore dans les pays jeunes ou en développement que dans les pays qui ont été développés. Les responsables politiques et notamment sanitaires et sociaux s'inquiètent. La gérontologie émerge de toute part. La gériatrie aussi avec cette paradoxale résistance des facultés de médecine à travers le monde, porteuses sans exception des pouvoirs liés au paradigme hippocratique, celui qui met au centre le diagnostic et le traitement correspondant. Un paradigme est un concept admis par tous sans avoir été démontré. Les gériatres reconnaissent le paradigme hippocratique comme essentiel pour en avoir tant fait bénéficier les patients dont ils ont la charge, mais ce paradigme pour indispensable n'est plus le seul espace des idées et des actions médicales gériatriques. Il faut en effet aujourd'hui savoir partager au sein de l'espace fonctionnel, gérontogériatrique, unissant les $2 \mathrm{G}$. Espace paradigmatique qui justifie seul et valorise toute l'action gériatrique, construite sur l'excellence du savoir médical mais s'exprimant aujourd'hui face à des aînés dont les demandes sont exclusivement celles du fonctionnement $\mathrm{du}$ corps, de l'esprit, de l'âme et du lien avec les autres. Aînés le plus souvent en santé et qui nous demandent de le rester, ou parfois fragiles ou malades et qui nous demandent plus que de guérir, de rester actifs sur le navire de la planète. Et là, dans ce paradigme fonctionnel, le pouvoir est partagé entre médecins, patients, familles, professionnels de santé, de l'aide, de l'éducation, technologues, gestionnaires et décideurs de toute catégorie, dont le poids politique représente le meilleur atout. Nous savons partager, nous gérontologues et gériatres. Nous avons sans doute su le faire avant les autres. Dans ce sens, la gérontogériatrie est certainement une valeur sûre.

Mais c'est sans doute au nom des droits des aînés, droits de l'Homme exercés chez et pour les aînés que nous aurons à justifier nos actions, nos recherches, nos enseignements, nos soins, nos aides et nos solidarités. La France les a vus naître

\section{A. Franco $(\bowtie)$}

Service de médecine interne et gériatrie, université de Nice

Sophia-Antipolis CHU, 06003 Nice cedex, France

e-mail : franco.a@chu-nice.fr il y a plus de 200 ans. Puis elle a un peu tardé à les soutenir au sein des Nations Unies. Tout laisse à croire qu'elle sera au rendez-vous avec les pays de la francophonie et aussi dans le concert planétaire de l'IAGG, pour le soutien des droits des aînés lors de la nécessaire élaboration d'un traité international des Nations Unies, droits que le quart, bientôt, de la population aura besoin d'exercer. Bien sûr, les pays développés peuvent donner un sens à ces droits, nécessaires au sein de leurs sociétés essentiellement démocratiques. Pourtant dans des pays qui risquent d'être vieux avant d'avoir connu la richesse ces droits sont vitaux. Nous évoquerons le traitement des maladies fréquentes au cours du vieillissement, nous aborderons les questions culturelles et éthiques du vieillissement et nous envisagerons comment les technologies peuvent apporter au citoyen vieillissant et à leur famille une meilleure qualité de vie. Mais dans certains coins de la planète, on tue encore hypocritement des vieilles, démentes ou non, pour sorcellerie, et en réalité pour récupérer leurs biens dès qu'elles sont veuves.

Le Congrès international francophone de gériatrie et gérontologie (CIFGG) Nice 2010 arrive à point nommé. Carrefour des idées avancées et événement international, il permettra de confronter les cultures, les sciences, les savoirs et leur transmission, les expériences professionnelles au sein de ce vaste espace culturel international francophone. Comme tous les quatre ans depuis 1978, c'est la neuvième fois que la planète gérontologique et gériatrique francophone se retrouvera au cours du CIFGG, porté par l'Association internationale francophone de gériatrie et gérontologie (IAGG) et organisé cette année sous l'égide de la Société française de gériatrie et gérontologie par un comité local d'organisation de collègues niçois.

Nice nous accueille du 19 au 21 octobre 2010 pour un rassemblement professionnel, scientifique et convivial dont les thèmes principaux seront :

- la Méditerranée, carrefour de la gérontologie ;

- la thérapeutique en gériatrie ;

- les gérontechnologies au service du soutien à domicile.

Communications libres ou thématiques, orales et affichées mettront l'accent sur l'interactivité entre les nombreux 
métiers, les nombreuses pratiques et les nombreuses cultures qui font l'intérêt et soulignent la modernité de nos belles disciplines au service des plus âgés.

Le volume de l'année gérontologique dédié au CIFGG en est l'expression. Un grand merci à son rédacteur en chef, le Pr Bruno Vellas, président de l'IAGG.

Merci à tous ceux qui ont contribué à cet événement et à son inscription dans la mémoire. Ces remerciements vont en premier lieu aux participants du CIFGG, conférenciers et auditeurs, garants de la valeur scientifique de l'événement. Merci au bureau de la gérontologie-gériatrie francophone représentée par l'IAGG, au soutien actif et sans faille de la Société française de gériatrie et gérontologie, aux sociétés scientifiques amies des pays francophones, dont plusieurs ont annulé leur congrès national en faveur de la rencontre de Nice, aux gériatres et gérontologues qui rejoignent notre communauté depuis l'Europe, le Moyen-Orient, l'Afrique et l'Amérique. Un grand et sincère merci au comité scientifique mis à contribution pour le programme, la sélection des communications et l'animation scientifique de l'événement, ainsi qu'à notre fidèle et actif comité local d'organisation qui travaille depuis quatre ans bientôt en lien avec notre organisatrice professionnelle et à tous ceux que je n'ai pas cités. Merci enfin à tous les soutiens du congrès, le secrétariat international à la Francophonie, le gouvernement français, la mairie de Nice, la communauté urbaine et le conseil général des Alpes-Maritimes.

Bienvenue à Nice. Nous vous attendons depuis quatre ans avec le plus grand plaisir de communiquer et d'apprendre. 